\title{
AMP-activated protein kinase $\alpha 1$ serves a carcinogenic role via regulation of vascular endothelial growth factor expression in patients with non-small cell lung cancer
}

\author{
GONG DAO HUI ${ }^{1}$, WANG YU XIU ${ }^{1 *}$, CHEN YONG $^{1}$, CHI BEI YUAN $^{1}$, ZHANG JUN $^{1}$, \\ GU JIAN JUN ${ }^{1}$, YANG JUN JUN ${ }^{1}$, XU XING XIANG ${ }^{1}$, HU SU WEI ${ }^{2,3}$ and MIN LING FENG ${ }^{1}$
}

${ }^{1}$ Department of Respiratory Medicine, Subei People's Hospital, Clinical Medical College of Yangzhou University, Yangzhou, Jiangsu 225001; ${ }^{2}$ Medical Genetic Center, The Affiliated Hospital of Yangzhou University, Yangzhou Women and Children's Hospital, Yangzhou, Jiangsu 225002; ${ }^{3}$ Department of Obstetrics and Gynecology, Nanjing Drum Tower Hospital, The Affiliated Hospital of Nanjing University Medicial School, Nanjing, Jiangsu 210008, P.R. China

Received May 9, 2018; Accepted February 4, 2019

DOI: $10.3892 / 01.2019 .10126$

\begin{abstract}
AMP-activated protein kinase $\alpha 1$ (AMPK $\alpha 1$ ) is involved in the tumorigenesis of various cancer types. However, the role of AMPK $\alpha 1$ in non-small cell lung cancer (NSCLC) remains unclear. In the present study, 99 NSCLC tumor tissues and paired normal tissues were obtained. The expression levels of AMPK $\alpha 1$ were significantly upregulated in NSCLC tumor tissues compared with those in adjacent non-tumor lung tissues. The patients were further divided into two groups according to their expression levels of AMPK $\alpha 1$ in tumor tissues. The results outlined that overexpression of AMPK $\alpha 1$ was associated with poor prognosis. In addition, vascular endothelial growth factor (VEGF) expression levels were associated with malignant progression in patients with NSCLC. Patients with NSCLC that overexpressed AMPK $\alpha 1$ and VEGF had the worst outcomes. Moreover, AMPK $\alpha 1$ may positively regulate VEGF expression. These results suggest that AMPK $\alpha 1$ serves a carcinogenic role at least in
\end{abstract}

Correspondence to: Professor Hu Su Wei, Department of Obstetrics and Gynecology, Nanjing Drum Tower Hospital, The Affiliated Hospital of Nanjing University Medicial School, 321 Zhongshan Road, Gulou, Nanjing, Jiangsu 210008, P.R China

E-mail: husuwei2004@126.com

Professor Min Ling Feng, Department of Respiratory Medicine, Subei People's Hospital, Clinical Medical College of Yangzhou University, Yangzhou, Jiangsu 225001, P.R. China

E-mail: minlingfeng@126.com

*Contributed equally

Abbreviations: AMPK $\alpha 1$, AMP-activated protein kinase $\alpha 1$; VEGF, vascular endothelial growth factor; NSCLC, non-small cell lung cancer; OS, overall survival

Key words: AMPKa1, VEGF, non-small cell lung cancer part through the regulation of VEGF expression, and thus represents a potential treatment target in patients with NSCLC.

\section{Introduction}

Malignant cells are characterized by uncontrolled growth and distant metastasis. An abundant energy supply is the foundation for cancer cell growth and metastasis, and as a result, metabolism is enhanced in cancer cells. As the primary regulator of cellular energy, AMP-activated protein kinase (AMPK) not only serves an important role in energy metabolism, but is also involved in cancer biology. AMPK is involved in tumorigenesis via the promotion of epithelial mesenchymal transition (1), regulation of cell growth and maintenance of tumor cell survival in numerous types of cancer. AMPK also has the potential to be a therapeutic target for cancer treatment (2,3). However, whether AMPK acts as an oncogene to promote cancer initiation and progression or a tumor suppressor is a matter of debate.

AMPK is a trimeric protein, and each subunit or isoform may have particular functions in different cancer types, which may account for the paradoxical effects of AMPK during tumorigenesis. Thus, it is necessary to demonstrate the role and mechanism of the AMPK isoforms separately. AMPK $\alpha 1$ (also known as protein kinase AMP-activated catalytic subunit $\alpha 1$ and encoded by the PRKAAl gene), is the most catalytic isoform of AMPK, and is predominantly expressed in the cytoplasm (4). Reportedly, the expression level of AMPK $\alpha 1$ was markedly increased in cancerous tissues compared with paired adjacent normal tissues, and the protein was preferentially expressed in the less differentiated areas of tumors (5). Furthermore, the proliferative ability of tumors was suppressed when AMPK $\alpha 1$ was inhibited (6). These findings indicated an oncogenic role for AMPK $\alpha 1$.

The present study was undertaken to investigate the role of AMPK $\alpha 1$ in non-small cell lung cancer (NSCLC). Tissue microarrays were used to determine the expression level of AMPK $\alpha 1$ in tumor tissues and adjacent normal tissues. Furthermore, lentiviruses were used to modulate the 
expression level of AMPK $\alpha 1$ in vitro. This aimed to determine whether AMPK $\alpha 1$ acts in a malignant manner in NSCLC, and ultimately the target of AMPK $\alpha 1$

\section{Materials and methods}

Patients and tissue samples. A total of 99 clinical NSCLC specimens were assessed. The tumor tissues and matched adjacent non-tumor tissues were collected from patients who had undergone surgical treatment at Subei People's Hospital (Yangzhou, China) between May 2004 and October 2012.

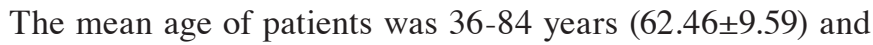
patient information is presented in Table I. The present study was approved by the ethics board of Subei People's Hospital, and informed consent was obtained from all participants. Fresh samples were paraffin-embedded and verified by pathological examination following hematoxylin and eosin staining. Tissues were fixed with $10 \%$ formalin for $24 \mathrm{~h}$ at room temperature and paraffin embedded. Samples were sliced into $4-\mu \mathrm{m}$ sections, which were stained with hematoxylin and eosin at room temperature for 10 and $2 \mathrm{~min}$, respectively. Samples were examined by two pathologists who had no prior knowledge of the patients' clinicopathological information, using a light microscope in three fields and with a x400 magnification.

Cell culture. The human lung adenocarcinoma cell line A549 (Yingrun Biotechnologies Inc., Changsha, China) was used for experiments in vitro. Parental A549 cells and lentivirus-transfected cells were cultured in RPMI-1640 medium supplemented with $10 \%$ fetal bovine serum (Biological Industries, Kibbutz Beit Haemek, Israel). Cells were maintained at $37^{\circ} \mathrm{C}$ in a humidified atmosphere $\left(5 \% \mathrm{CO}_{2}\right)$.

Tissue microarray (TMA) and immunohistochemistry (IHC). A total of 99 pairs of NSCLC tumor tissue samples and adjacent non-tumor tissue samples were fixed with $10 \%$ formalin for $24 \mathrm{~h}$ at room temperature and paraffin embedded to obtain the TMAs. Using biopsy needles, tissue cores (diameter, $3 \mathrm{~mm}$ ) were obtained and placed on a recipient block. IHC was carried out using streptavidin-biotin-peroxidase complex method. TMA slides for IHC were deparaffinized by bathing in xylene solution for $10 \mathrm{~min}$, and rehydrated through decreasing ethanol gradient (100, 90, 75 and 50\%) for 5 min. Antigen retrieval was performed by incubating slides with citrate buffer (pH 6.0) at $95^{\circ} \mathrm{C}$ for $15 \mathrm{~min}$. Endogenous peroxidase activity was blocked by incubating slides in $3 \%$ $\mathrm{H}_{2} \mathrm{O}_{2}$ at room temperature for $15 \mathrm{~min}$. The slides were then incubated with primary antibodies against AMPK $\alpha 1$ (1:100; cat. no. D63G4; Cell Signaling Technology, Inc., Danvers, MA, USA) and VEGFA (1:100; cat. no. WL00009b; Wanleibio Co., Ltd., Shanghai, China) overnight at $4^{\circ} \mathrm{C}$. The slides were incubated with biotin-labeled anti-rabbit secondary antibodies (1:100; cat. no. A0279; Beyotime Institute of Biotechnology, Haimen, China) at room temperature for $30 \mathrm{~min}$, and detected with 3,3-diaminobenzidine (1:300; cat. no. P0203; Beyotime Institute of Biotechnology). Staining intensity and extent were examined using a light microscope at $\mathrm{x} 400$ magnification. Staining intensity was scored as $0,1,2$ or 3 for negative, weak, moderate or strong staining, respectively. The extent of staining was scored based on the percentage of AMPK $\alpha 1$ - or
Table I. Clinicopathological characteristics of patients with non-small cell lung cancer.

\begin{tabular}{lcc}
\hline Variables & Cases, $\mathrm{n}$ & Cases, $\%$ \\
\hline Age (years) & & \\
$<60$ & 37 & 34.3 \\
$\geq 60$ & 62 & 65.7 \\
Sex & & \\
Male & 73 & 73.7 \\
Female & 26 & 26.3 \\
Histotype & & \\
Adenocarcinoma & 51 & 51.5 \\
Squamous cell & 48 & 48.5 \\
Primary tumor & & \\
T1 & 21 & 21.2 \\
T2 & 54 & 54.5 \\
T3 & 17 & 17.2 \\
T4 & 7 & 7.1 \\
Regional lymph node & & \\
N0 & 67 & 67.7 \\
N1 & 19 & 19.2 \\
N2 & 11 & 11.1 \\
N3 & 2 & 2.0 \\
Tumor-node-metastasis stage & & \\
I-II & 58 & 58.6 \\
III-IV & 41 & 41.4 \\
\hline
\end{tabular}

VEGF-positive tumor cells. The final score was obtained by summing the scores for staining intensity and extent of staining.

Lentivirus and cell infection. To modulate AMPK $\alpha 1$ expression in vitro, lentiviruses, including LV-PRKAA1-RNAi (expressing a short interfering RNA targeting the PRKAAI gene; 5'-CTTTGCTTCTCTTATAAGTTATTGTGA-3'), LV-PRKAA1 (a lentivirus overexpressing the PRKAAl gene), and a negative LV-control were purchased from Shanghai GeneChem Co., Ltd. (Shanghai, China). A549 cells were infected with lentivirus according to the manufacturer's protocol. Briefly, $5 \times 10^{4}$ cells supplemented with lentivirus (multiplicity of infection, 10) and $1 \mu 1$ Polybrene (Shanghai GeneChem Co., Ltd.) were grown on 24-well plates. The supernatant was removed after $24 \mathrm{~h}$ and fresh culture medium was added to the cells. Fluorescence microscopy was used to observe the infection rate $72 \mathrm{~h}$ postinfection, and stable clones were selected after 2 weeks using puromycin $(2 \mu \mathrm{g} / \mathrm{ml})$ (GE Healthcare Life Sciences, Little Chalfont, UK). Western blotting was conducted to confirm the final infection efficiency.

Western blotting. Cells were harvested and lysed with radioimmunoprecipitation assay buffer (cat. no. P0013B; Beyotime Institute of Biotechnology) and the protein concentration was measured using a bicinchoninic acid assay. Sample concentrations were equalized, and proteins ( $29 \mu \mathrm{g}$, Fig. 4A; 
$15 \mu \mathrm{g}$, Fig. 4B) were separated by SDS-PAGE (10\% gels). The proteins were subsequently transferred to polyvinylidene fluoride membranes and blocked with 5\% skimmed milk in Tris-buffered saline for $2 \mathrm{~h}$ at room temperature. The membranes were incubated with primary antibodies against AMPK $\alpha 1$ (1:1,000; cat. no. D63G4; Cell Signaling Technology, Inc.) and vascular endothelial growth factor A (VEGFA; 1:500; cat. no. WL00009b; Wanleibio Co., Ltd) overnight at $4^{\circ} \mathrm{C}$, prior to incubation with a secondary horseradish peroxidase-labeled goat anti-rabbit antibody (1:1,000; cat. no. KGAA35; Nanjing KeyGen Biotech Co., Ltd., Nanjing, China) for $1 \mathrm{~h}$ at room temperature. Bands were visualized using BeyoECL Plus (Beyotime Institute of Biotechnology). The primary antibody against GAPDH, (1:1,000, cat. no. 14C10; Cell Signaling Technology, Inc.) was used as an internal control. Densitometric analysis was performed using Image $\mathrm{J}$ v1.50i Software (National Institutes of Health, Bethesda, MD, USA).

Statistical analysis. Statistical analysis was performed using SPSS 22.0 (IBM Corp., Armonk, NY, USA). Data were expressed as the mean \pm standard deviation, and analyzed using the Student's t-test. A Chi-square test was used to evaluate the association between AMPK $\alpha 1$ and VEGF. Kaplan-Meier curves were generated to assess overall survival (OS). Patients were classified based on the AMPK $\alpha 1$ or VEGF expression level in their tumor tissue into high expression $(\geq$ median; $\mathrm{n}=49$ ) or low expression ( $<$ median; $\mathrm{n}=50$ ) groups. $\mathrm{P}<0.05$ was considered to indicate a statistically significant difference.

\section{Results}

Patients characteristics. The characteristics of the 99 surgically resected NSCLC tumors (51 adenocarcinoma and 48 squamous cell carcinoma samples) are displayed in Table I. Elderly patients ( $\geq 60$ years old) accounted for $65.7 \%$, of which 73 were male $(73.7 \%)$ and 26 female $(26.3 \%)$. The proportion of patients with early and late stage NSCLC was 58.6 and $41.4 \%$, respectively.

High expression of AMPK $\alpha 1$ is associated with poor prognosis in NSCLC. The expression levels of AMPK $\alpha 1$ in NSCLC tumor tissues were compared with those in adjacent non-tumor lung tissues; AMPK $\alpha 1$ expression levels were significantly higher in tumor tissues compared with those in the adjacent tissues (Fig. 1A). The Kaplan-Meier method was used to determine the association between AMPK $\alpha 1$ expression level and prognosis. Patients with high AMPK $\alpha 1$ expression levels had a shorter OS time compared with patients with low AMPK $\alpha 1$ levels (Fig. 1B). According to these results, AMPK $\alpha 1$ may serve an aggressive role in NSCLC, and high levels of AMPK $\alpha 1$ may act as an indicator of poor prognosis in patients with NSCLC.

VEGF expression levels are associated with prognosis in patients with NSCLC. Angiogenesis has a prominent role during cancer occurrence, development, and metastasis. VEGF, an angiogenesis-driving factor, influences malignancy and is associated with poor prognosis in various types of cancer $(7,8)$. Therefore, the association between VEGF and AMPK $\alpha 1$ levels in NSCLC were investigated. Consistent with previous reports $(9,10)$, it was concluded that VEGF was more
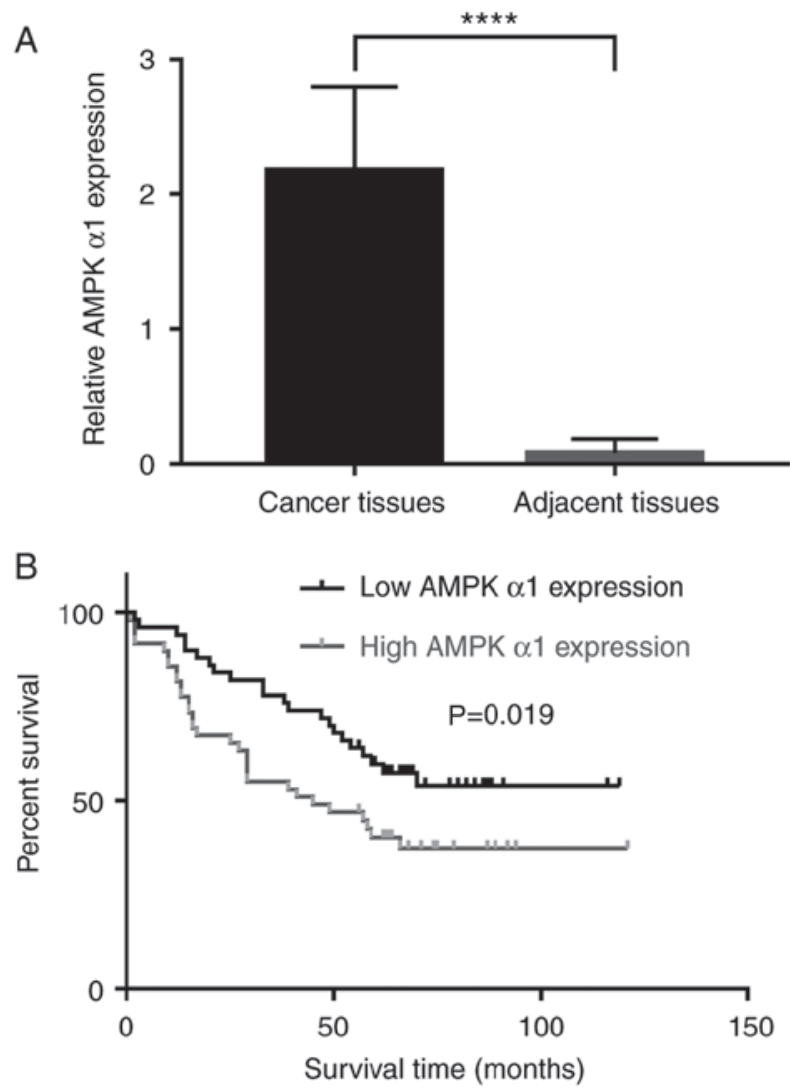

Figure 1. Expression level of AMPK $\alpha 1$ is upregulated in cancerous tissues and associated with poor prognosis. (A) Compared with that in non-tumor lung tissues, the expression level of AMPK $\alpha 1$ was significantly upregulated in cancer tissues. (B) Patients with high expression levels of AMPK $\alpha 1$ had a shorter overall survival compared with that of the low expression group. Data were expressed as the mean \pm standard deviation. ${ }^{* * * *} \mathrm{P}<0.0001$. AMPK $\alpha 1$, AMP-activated protein kinase $\alpha 1$.

highly expressed in NSCLC tissues compared with adjacent non-tumor lung tissues (Fig. 2A), and higher levels of VEGF were associated with a poorer prognosis (Fig. 2B).

Patients with NSCLC that express high levels of AMPK $\alpha 1$ and VEGF have a shorter OS. Based on the conclusion that AMPK $\alpha 1$ and VEGF are associated with NSCLC, patients that expressed high levels of AMPK $\alpha 1$ and VEGF ( $n=31$; Table II) were selected and further classified. Patients that expressed high levels of AMPK $\alpha 1$ and VEGF had a significantly shorter OS compared with those that expressed low levels of AMPK $\alpha 1$ and VEGF, and those with all other expression level combinations (AMPK $\alpha 1$ low, VEGF low; AMPK $\alpha 1$ high, VEGF low; and AMPK $\alpha 1$ low, VEGF high; Fig. 3A and B).

AMPK al positively regulates VEGF expression in NSCLC in vitro. The potential regulatory association between AMPK $\alpha 1$ and VEGF in NSCLC was investigated. Western blotting was performed to detect the effects AMPK $\alpha 1$ expression level on that of VEGF. As illustrated in Fig. 4A, downregulation of AMPK $\alpha 1$ resulted in decreased VEGFA protein expression in A549 cells, and upregulation of AMPK $\alpha 1$ resulted in increased VEGFA protein expression (Fig. 4B). The results indicated that AMPK $\alpha 1$ positively regulates VEGF expression in NSCLC. 
A

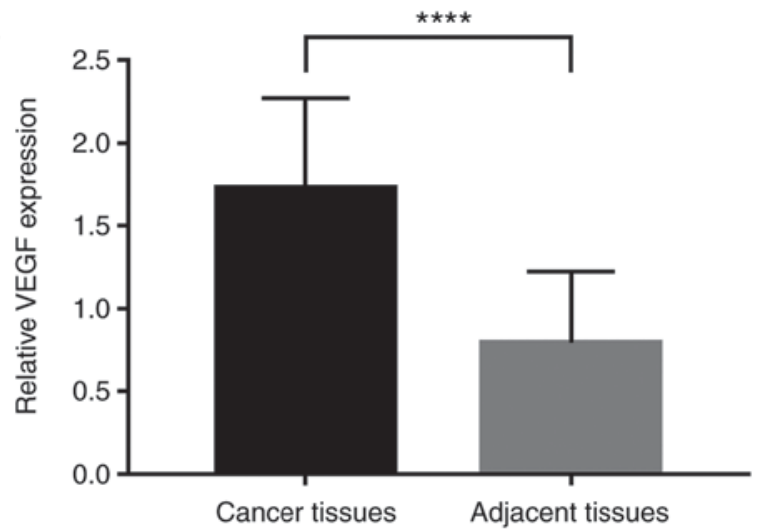

B

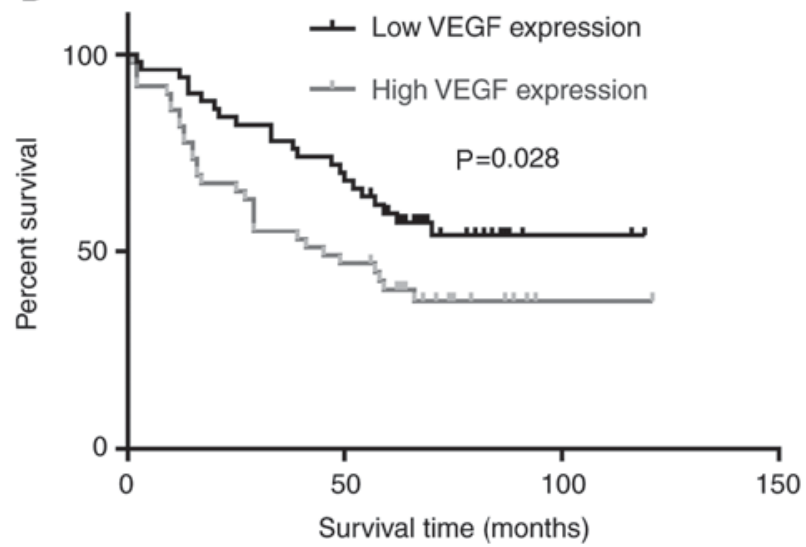

Figure 2. Increased expression of VEGF is associated with poor prognosis in non-small cell lung cancer. (A) Compared with that in non-tumor lung tissues, the expression level of VEGF was significantly upregulated in cancer tissues. (B) Patients with a high expression level of VEGF had a shorter overall survival compared with that of the low expression group. Data were expressed as the mean \pm standard deviation. ${ }^{* * * *} \mathrm{P}<0.0001$. VEGF, vascular endothelial growth factor.

\section{Discussion}

Lung cancer is a commonly occurring malignancy with high mortality rates worldwide (11). Based on cell morphology, lung cancer is predominantly divided into two types: NSCLC and small cell lung cancer (SCLC), which account for 80 and $20 \%$ of all lung cancer cases, respectively. Although the detection rate and treatment have improved over time, the clinical outcomes of patients with lung cancer remain unsatisfactory $(12,13)$. In addition, compared with SCLC, patients with NSCLC are less sensitive to chemotherapy. To achieve an improved outcome for patients with NSCLC, further studies to understand the mechanisms underlying tumorigenesis and metastasis, and the development effective treatments are required.

In the present study it was demonstrated that AMPK $\alpha 1$ was highly expressed in tumor tissues compared with non-tumor lung tissues, and overexpression of AMPK $\alpha 1$ was negatively associated with prognosis in patients with NSCLC. This observation of the aggressive role of AMPK $\alpha 1$ in NSCLC was consistent with previous findings $(6,14)$. However, AMPK $\alpha 1$ has also been indicated as a tumor suppressor (15), and its deletion reportedly promotes cell proliferation and angiogenesis (16). This discrepancy in
Table II. Association between the expression levels of AMPK $\alpha 1$ and VEGF.

\begin{tabular}{lccc}
\hline & \multicolumn{2}{c}{$\begin{array}{c}\text { AMPK } \\
\alpha 1 \text { expression }\end{array}$} & \\
\cline { 2 - 3 } VEGF expression & High & Low & P-value \\
\hline High & 31 & 18 & 0.006 \\
Low & 18 & 32 & \\
\hline
\end{tabular}

AMPK $\alpha 1$, AMP-activated protein kinase $\alpha 1$; VEGF, vascular endothelial growth.
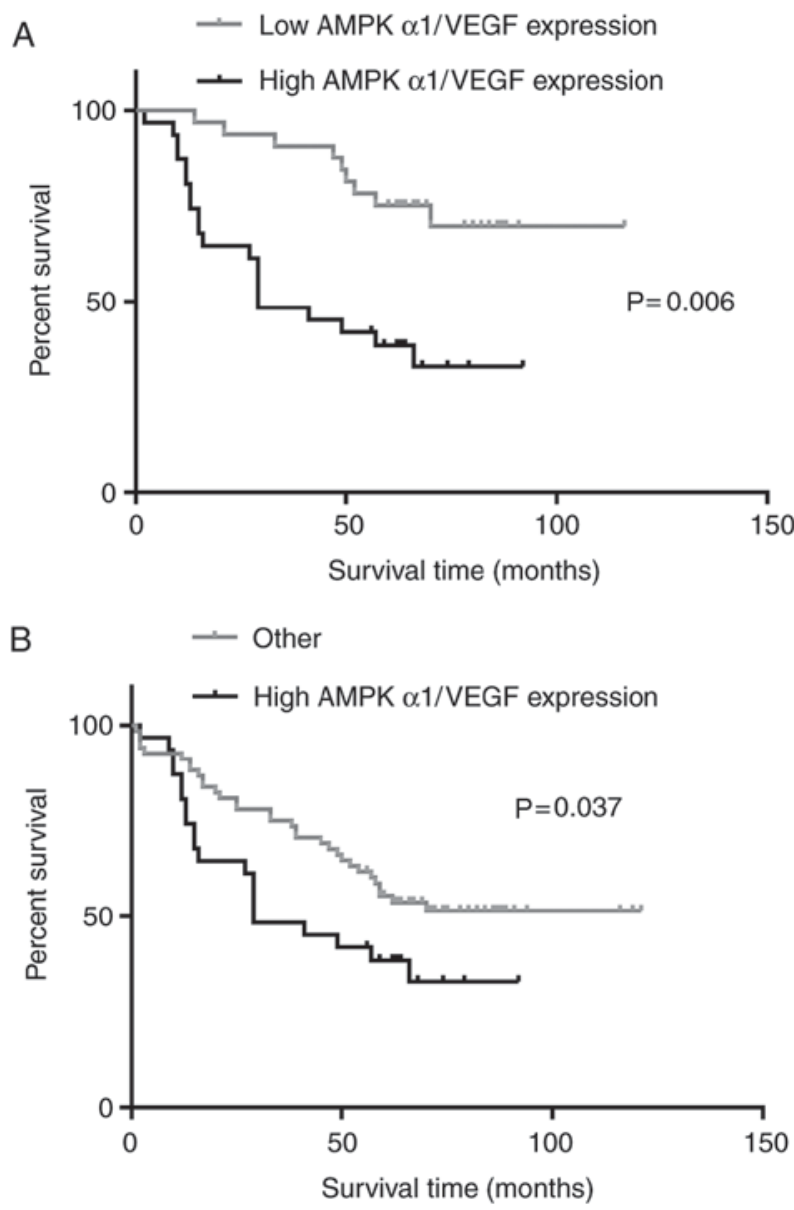

Figure 3. Patients with high expression of AMPK $\alpha 1$ and VEGF have shorter OS. (A) Compared with patients expressing low levels of AMPK $\alpha 1$ and VEGF, patients expressing high levels of these proteins exhibited a significantly shorter OS. (B) Compared with the remaining groups of patients (AMPK $\alpha 1$ low, VEGF low; AMPK $\alpha 1$ high, VEGF low; and AMPK $\alpha 1$ low, VEGF high), patients with NSCLC expressing high levels of AMPK $\alpha 1$ and VEGF displayed a significantly shorter OS. OS, overall survival; AMPK $\alpha 1$, AMP-activated protein kinase $\alpha 1$; VEGF, vascular endothelial growth factor; NSCLC, non-small cell lung cancer.

different cancer types may be the result of different tissue systems, in addition to different targets and associated pathways. Furthermore, it was revealed that AMPK $\alpha 1$ positively regulated VEGF expression. Considering that VEGF is a well-known tumor driver gene, it was hypothesized that the 
A
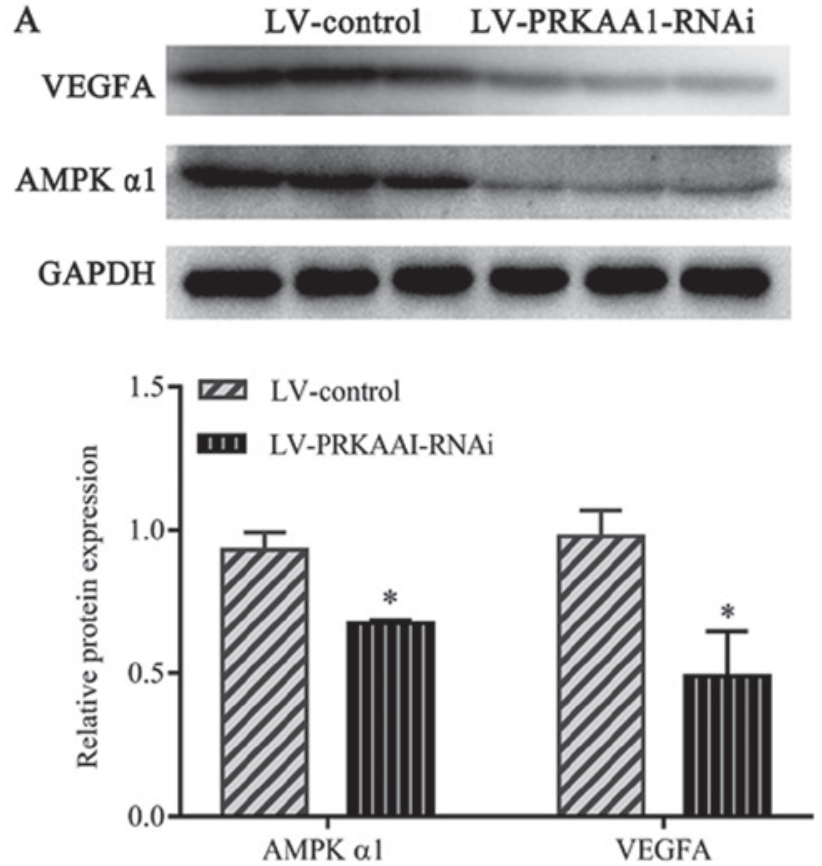

B
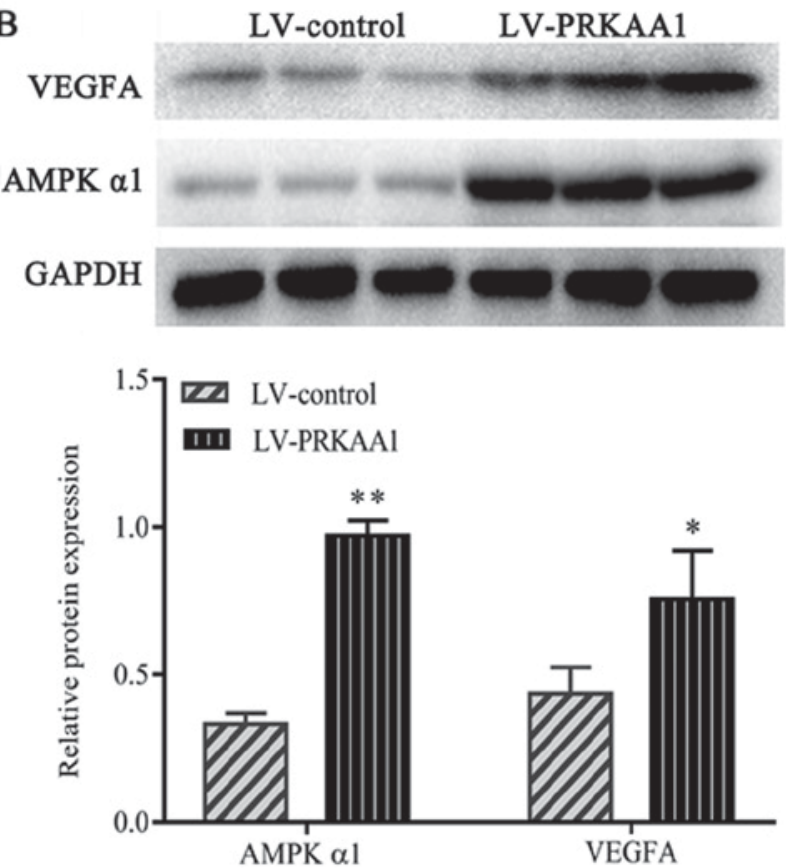

Figure 4. AMPK $\alpha 1$ positively regulates VEGF expression in NSCLC in vitro. (A) Downregulation of AMPK $\alpha 1$ decreased VEGFA protein expression levels in A549 cells. "P<0.05 vs. LV-control group. (B) Upregulation of AMPK $\alpha 1$ led to increased VEGFA protein expression levels. " $\mathrm{P}<0.05$, ${ }^{* *} \mathrm{P}<0.01$ vs. LV-control group. LV-PRKAA1-RNAi, lentivirus expressing a short interfering RNA targeting PRKAA1 (the gene encoding AMPK $\alpha 1$ ); LV-PRKAA1, lentivirus overexpressing PRKAA1. LV, lentivirus; AMPK $\alpha 1$, AMP-activated protein kinase $\alpha 1$; VEGF, vascular endothelial growth factor.

malignant role of AMPK $\alpha 1$ may be influenced by the regulation of VEGF expression in NSCLC. Anti-angiogenesis of cancer cells is a promising method to treat cancer. However to date, its efficacy has been limited (17). In the present study, a positive regulatory association was observed between AMPK $\alpha 1$ and VEGF, such that patients possessing tumors with high levels of these proteins had poorer clinical outcomes. Therefore, simultaneous targeting of AMPK $\alpha 1$ and VEGF may enhance the anti-cancer efficacy of anti-VEGF therapy.

The present study was limited in the following ways: Firstly, this was a retrospective, single institution study, and the number of specimens used to analyze the association between AMPK $\alpha 1$ and prognosis was small. Moreover, the exact mechanism of AMPK $\alpha 1$ regulation of VEGF in NSCLC was not investigated.

In conclusion, the present study identified an aggressive pro-cancerous effect of high levels of AMPK $\alpha 1$ in patients with NSCLC. A regulatory association between AMPK $\alpha 1$ and VEGF was also demonstrated. The results indicated that AMPK $\alpha 1$ is a potential biomarker and therapeutic target in NSCLC, which requires further investigation in vitro and in vivo.

\section{Acknowledgements}

Not applicable

\section{Funding}

The present study was supported by the National Natural Science Foundation of China (grant no. 81870033, 81302016 and 81302015), the National Natural Science Foundation of Jiangsu Province (grant nos. BK20130456 and BK20140101) and the Six Talent Peaks Project of Jiangsu Province (grant no. WSN-106).

\section{Availability of data and materials}

The datasets used during the current study are available from the corresponding author on reasonable request.

\section{Authors' contributions}

XXX, HSW and MLF made contributions to conception and design of this study. WYX, CY, CBY, ZJ, GJJ and YJJ analyzed and interpreted the patients' data. GDH contributed to the conception and the design of this study and wrote the manuscript. All authors read and approved the final manuscript.

\section{Ethics approval and consent to participate}

The present study was approved by the Ethics Board of Subei People's Hospital (Yangzhou, China), and written informed consent was obtained from all participants.

\section{Patient consent for publication}

All of the patients provided written informed consent for the publication of any associated data.

\section{Competing interests}

The authors declare that they have no competing interests. 


\section{References}

1. Cazarin JM, Coelho RG, Hecht F, Andrade BM and Carvalho DP: 5'-AMP-activated protein kinase regulates papillary (TPC-1 and BCPAP) thyroid cancer cell survival, migration, invasion, and epithelial-to-mesenchymal transition. Thyroid 26: 933-942, 2016

2. Zou J, Hong L, Luo C, Li Z, Zhu Y, Huang T, Zhang Y, Yuan H, $\mathrm{Hu} \mathrm{Y}$, Wen T, et al: Metformin inhibits estrogen-dependent endometrial cancer cell growth by activating the AMPK-FOXO1 signal pathway. Cancer Sci 107: 1806-1817, 2016.

3. Zadra G, Photopoulos C, Tyekucheva S, Heidari P, Weng QP, Fedele G, Liu H, Scaglia N, Priolo C, Sicinska E, et al: A novel direct activator of AMPK inhibits prostate cancer growth by blocking lipogenesis. EMBO Mol Med 6: 519-538, 2014.

4. Choi CH, Chung JY, Cho H, Kitano H, Chang E, Ylaya K, Chung EJ, Kim JH and Hewitt SM: Prognostic significance of AMP-dependent kinase alpha expression in cervical cancer. Pathobiology 82: 203-2011, 2015.

5. Huang FY, Chiu PM, Tam KF, Kwok YK, Lau ET, Tang MH, Ng TY, Liu VW, Cheung AN and Ngan HY: Semi-quantitative fluorescent PCR analysis identifies PRKAA1 on chromosome 5 as a potential candidate cancer gene of cervical cancer. Gynecol Oncol 103: 219-225, 2006.

6. Laderoute KR, CalaoaganJM, Chao WR, Dinh D, DenkoN, Duellman S, Kalra J, Liu XH, Papandreou I, Sambucetti L and Boros LG: Experimental human breast cancer supports the growth of aggressive 5'-AMP-activated protein kinase (AMPK). J Biol Chem 289: 22850-22864, 2014.

7. Lin Y, Liu F, Fan Y, Qian X, Lang R, Gu F, Gu J and Fu L: Both high expression of pyruvate kinase $\mathrm{M} 2$ and vascular endothelial growth factor-C predicts poorer prognosis in human breast cancer. Int J Clin Exp Pathol 8: 8028-8037, 2015.

8. Yalniz Z, Tigli H, Tigli H, Sanli O, Dalay N and Buyru N: Novel mutations and role of the LKB1 gene as a tumor suppressor in renal cell carcinoma. Tumour Biol 35: 12361-12368, 2014.

9. Yuan Y, Min SJ, Xu DQ, Shen Y, Yan HY, Wang Y, Wang W and Tan YJ: Expressions of VEGF and miR-21 in tumor tissues of cervical cancer patients with HPV infection and their relationships with prognosis. Eur Rev Med Pharmacol Sci 22: 6274-6279, 2018.
10. Wang F, Peng L, Wang Y and Liu X: A Meta-analysis of vascular endothelial growth factor for nasopharyngeal cancer prognosis. Front Oncol 8: 486, 2018.

11. Bray F, Ferlay J, Soerjomataram I, Siegel RL, Torre LA and Jemal A: Global cancer statistics 2018: GLOBOCAN estimates of incidence and mortality worldwide for 36 cancers in 185 countries. CA Cancer J Clin 68: 394-424, 2018.

12. Bagcchi S: Lung cancer survival only increases by a small amount despite recent treatment advances. Lancet Respir Med 5: 169, 2017.

13. Ettinger DS, Wood DE, Akerley W, Bazhenova LA, Borghaei H, Camidge DR, Cheney RT, Chirieac LR, D'Amico TA, Demmy TL, et al: Non-small cell lung cancer, version 6.2015 . J Natl Compr Canc Netw 13: 515-524, 2015.

14. Xu H, Zhou Y, Coughlan KA, Ding Y, Wang S, Wu Y, Song P and Zou M: AMPKa1 deficiency promotes cellular proliferation and DNA damage via p21 reduction in mouse embryonic fibroblasts. Biochim Biophys Acta 1853: 65-73, 2015.

15. Faubert B, Boily G, Izreig S, Griss T, Samborska B, Dong Z, Dupuy F, Chambers C, Fuerth BJ, Viollet B, et al: AMPK is a negative regulator of the Warburg effect and suppresses tumor growth in vivo. Cellmetab 17: 113-1124, 2013.

16. Zhou Y, Xu H, Ding Y, Lu Q, Zou MH and Song P: AMPKa1 deletion in fibroblasts promotes tumorigenesis in athymic nude mice by p52-mediated elevation of erythropoietin and CDK2. Oncotarget 7: 53654-53667, 2016.

17. Singh R, Kim WJ, Kim PH and Hong HJ: Combined blockade of HER2 and VEGF exerts greater growth inhibition of HER2-overexpressing gastric cancer xenografts than individual blockade. Exp Mol Med 45: e52, 2013.

This work is licensed under a Creative Commons

Attribution-NonCommercial-NoDerivatives 4.0 International (CC BY-NC-ND 4.0) License. 